\title{
ИНТЕЛЛЕКТУАЛЬНЫЕ СИСТЕМЫ
}

\section{UDC 004.85}

\author{
Mukhanov S.B.*, Aldanazar A.A., Uatbayeva A.M., Alimbekov A.Ye., Marat G.S. \\ International Information Technology University, Almaty
}

\section{COMPETITIVE LEARNING IN NEURAL NETWORKS}

\begin{abstract}
The article presents the basic concept of competitive learning in neural networks. Provides the main machine learning learning models and applications. The analysis of the advantages and disadvantages of these models is carried out. The geometric interpretation of competitive learning is presented in terms of mathematical formulas, as well as the behavior of neurons in this model. Neural systems are described as a powerful tool and driver in the field of modern Internet technologies, in data science and big (meta) data.
\end{abstract}

Key words: competitive learning, neural networks, learning, perceptron, synaptic weights.

\section{Introduction}

Today, the development of artificial neural networks creates favorable conditions for the formation of a single educational, scientific space. Neural networks are a versatile technology with a variety of disciplines such as neurophysiology, mathematics, physics, statistics, computer science and engineering [1]. They are used in various fields: medicine, business, industry, geology, artificial intelligence and others.

Artificial neural networks - train, create, research and develop intelligent systems these are modeling, machine learning, diagnostics, pattern recognition, information processing, etc.

Information has become the main, important value of modern society. The main attention of the state is paid to the development of digital technologies.

\section{Basic concepts}

In the modern world, digital technologies play an increasingly important role in the development of countries' economies.

Digital technologies have given a number of advantages - simplified access for the population and business to public services, accelerated information exchange, the emergence of new opportunities for doing business, the creation of new digital products, etc.

In modern Kazakhstan, the direction of applications of artificial neural networks is supported only in some areas. It is necessary to more actively develop the direction in the field of intelligent systems in order to achieve sustainable economic growth, for effective management of educational facilities and processes, increase the competitiveness of the economy, and improve the living standards of the population. It is also necessary to attract and train more specialists to develop software in various fields of activity, improve digital technologies, technological processes and improve information security protection.

Let's try to figure out what parameters are important in learning processes.

The most $[2,3]$ important property of neural networks is their ability to learn from environmental data and, as a result of training, improve their performance.

Learning is a process where free parameters of a neural network are set by simulating the environment in which the network is embedded. This type of learning is focused by adjusting these parameters. The description of this learning process has the following event steps:

1. The neural network receives stimuli from the external environment.

2. As a result, free parameters of the neural network change. 
3. After changing the internal structure, the neural network responds to excitations in a different way.

The above list of clear rules for solving a learning problem is called a learning algorithm. Learning algorithms differ from each other in the way they adjust the synaptic weights of neurons. Another distinctive characteristic is the way in which the trained neural network communicates with the outside world [4]. In this context, they talk about a learning paradigm associated with the model of the environment in which a given neural network functions.

There are five basic learning models

1. Based on error correction - big data (big data), data science

2. Learning based on memory - pattern recognition systems (semantic network)

3. Hebb's method - in scientific projects and laboratories

4. Boltzmann's method - in the field of statistics algorithms

Competitive learning - marketing, online shopping, electronic exchange.

\section{Competitive learning}

In competitive learning, the output neurons of the neural network compete with each other for the right to be activated; only one neuron can be active at a time. This property makes competitive learning useful for learning statistical properties. The competitive learning rule is based on three basic elements.

1. A set of identical neurons with randomly distributed synoptic weights, leading to different responses of neurons to the same input signal.

2. Limit value (Limit) "strength" of each neuron.

3. A mechanism that allows you to compete for the right to respond to a given subset of input signals and determines the only active output neuron. The winning neuron in this competition is called the winning neuron, and the principle of competitive learning is formulated as the slogan "winner takes everything" [5].

The simplest neural network with competitive learning contains a single layer of output neurons, each of which is connected to input nodes. In such a network, feedbacks between neurons can exist.

For neuron $\mathrm{k}$ to win the competition, its induced local field $\mathrm{v} \_\mathrm{k}$ for a given input image $\mathrm{x}$ must be the maximum among all neurons in the network. Then the output signal $\mathrm{y} \_\mathrm{k}$ of the winner neuron $\mathrm{k}$ is taken equal to one. The output signals of the remaining neurons are then set to zero. Thus, we can write:

$$
y_{k}=\left\{\begin{array}{c}
1, \text { if } v_{k},>v_{j} \text { for all } j, j \neq k, \\
0 \quad \text { in other cases, }
\end{array}\right.
$$

where the induced local field $v_{k}$ represents the combined excitation of neuron $\mathrm{k}$ from all input and feedback signals.

Let be $\omega_{k j}$ - the synaptic weight of the connection between the input node $\mathrm{j}$ and the neuron $\mathrm{k}$. Let us assume that the synaptic weights of all neurons are fixed, while

$$
\sum_{j} \omega_{k j}=1 \text { for all } k \text {. }
$$

Then the training of this neuron consists in shifting the synaptic weights from inactive to active input nodes. If a neuron does not form a response to a specific input image, then it does not learn $[7,8]$. If some neuron wins in the competition, then the weights of the connections of this neuron are evenly distributed between its active input nodes, and the connections with inactive input nodes are weakened. 

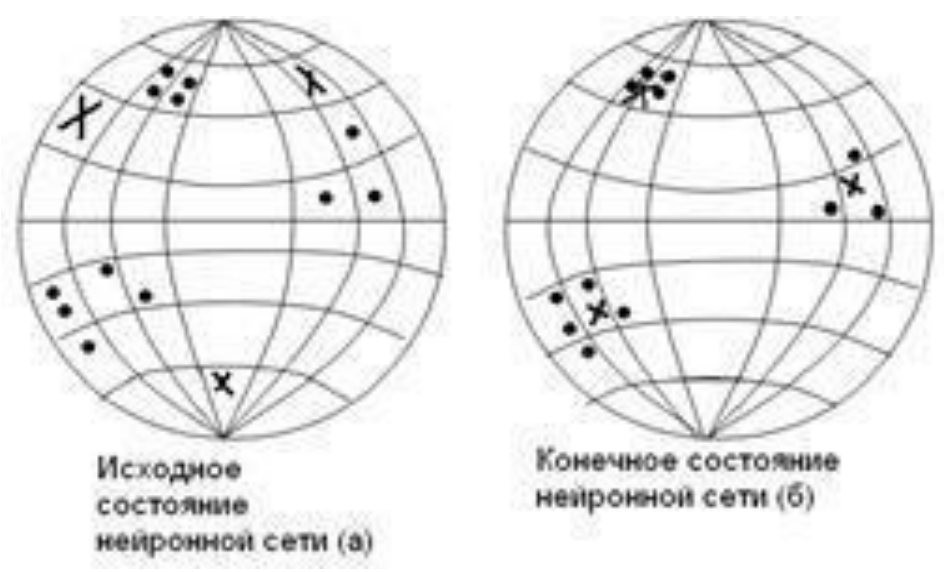

Figure 1 - Geometric interpretation of competitive learning

According to the competitive learning rule, change $\Delta_{\omega k j}$ synaptic weight $\omega_{k j}$ is defined by the following expression:

$$
{ }_{k j}^{\Delta \omega}=\left\{\begin{array}{l}
\eta\left(x_{j}-\omega_{k j}\right), \text { if neuron } k \text { wins the competition, } \\
0, \quad \text { if neuron } k \text { wins the competition, }
\end{array}\right.
$$

Where $\eta$ - learning rate parameter. This rule reflects the displacement of the vector of synaptic weight $\omega_{-} \mathrm{k}$ of the winning neuron $\mathrm{k}$ towards the input image $\mathrm{x}$.

A geometric analogy can be used to illustrate the essence of competitive learning. It is assumed that all input images (vectors) $\mathrm{x}$ have some constant Euclidean norm. Thus, they can be depicted as points on the $\mathrm{N}$-dimensional unit sphere, where $\mathrm{H}$ is the number of input nodes [9]. $\mathrm{N}$ is also the dimension of the vector of synaptic weights $\omega_{-} \mathrm{k}$. It is assumed that all neurons in the network have the same Euclidean length (norm), that is:

$$
\sum_{j} \omega_{k j}^{2}=1 \text { for all } k \text {. }
$$

When the synaptic weights are properly scaled, they form a set of vectors that are projected onto the same N-dimensional unit sphere. In Figure 1, a, three natural groups (clusters) of points can be distinguished, representing the input images. This figure also shows the likely initial state of the network (marked with crosses) prior to training. Figure 1b shows the final state of the network, obtained as a result of competitive learning, in which the synaptic weights of each input neuron are shifted to the centers of gravity of the corresponding clusters. This example demonstrates the ability of a neural network to solve clustering problems in the process of competitive learning. However, for a "stable" solution of this problem, the input images must form rather scattered groups of vectors. Otherwise, the network may become unstable, since responses from various output neurons will be formed in response to a given input image.

\section{Neural network systems}

Neural network architecture

ANN (Artificial Neural network) can be considered as a weighted directed graph, in which artificial neurons are nodes. According to the architecture of connections, ANNs can be grouped into two classes (Fig. 2): feedforward networks, in which graphs do not have loops, and recurrent networks, or networks with feedback. 


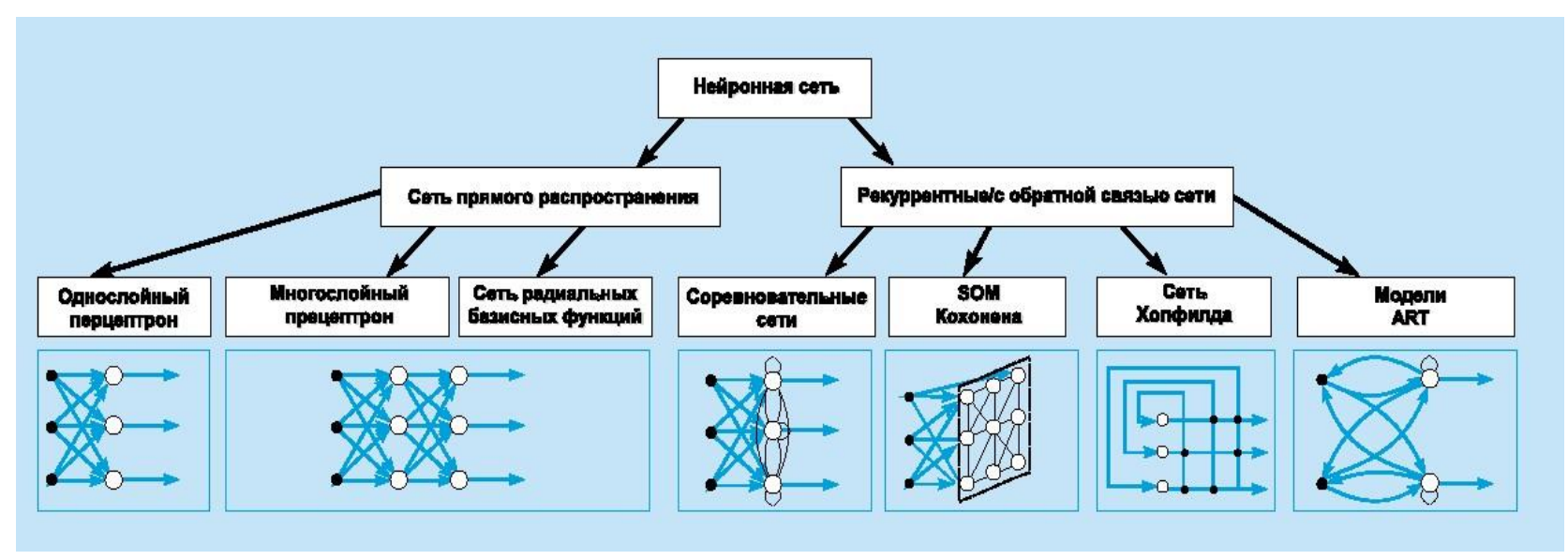

Figure 2 - Systematization of feedforward and recurrent network architectures (with feedback)

In the most common family of first-class networks, called the multilayer perceptron, neurons are arranged in layers and have unidirectional connections between layers. In fig. 2 shows typical networks of each class. Feedforward networks are static in the sense that, for a given input, they generate one set of output values that do not depend on the previous state of the network [10]. Recurrent networks are dynamic, since, due to feedbacks, the inputs of neurons are modified in them, which leads to a change in the state of the network.

\section{Multilayer feedforward networks}

A standard L-layer feedforward network consists of a layer of input nodes (we will adhere to the statement that it is not included in the network as an independent layer), (L-1) hidden layers and an output layer connected in series in the forward direction and not containing connections between elements within a layer and feedbacks between layers. In fig. 3 shows the structure of a three-layer network.

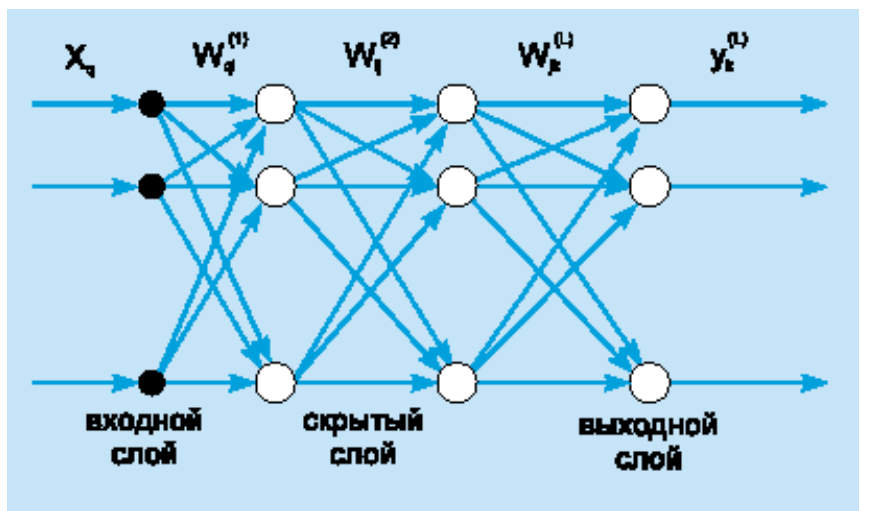

Figure 3 - Typical architecture of a three-layer feedforward network

\section{Multilayer perceptron}

The most popular class of multilayer feedforward networks is formed by multilayer perceptrons, in which each computational element uses a threshold or sigmoidal activation function. A multilayer perceptron can form arbitrarily complex decision boundaries and implement arbitrary Boolean functions [6]. The development of a backpropagation algorithm for determining weights in a multilayer perceptron made these networks the most popular among researchers and users of neural networks. Geometric interpretation [10] explains the role of elements of hidden layers (using the threshold activation function). 
For example, you can consider the application of competitive learning in the field of the Internet, more precisely in Web technologies. At the moment, over a million sites have been created, and they work on different technologies. Today, website developers have many choices as to which language or technology to use to build a website. Many developers use different programming languages and technologies in different projects for specific goals and objectives. There are many comparisons between software development tools, as well as their effective use and performance. The use of neural networks in this area is necessary to improve the mobility, performance and protection of sites from external attacks that violate network security and data confidentiality in an unauthorized way.

The future of the Internet largely depends on many technology factors, including automatic assembly based on artificial intelligence and machine learning.

\section{Conclusion}

The science of neural networks is a powerful tool for working with large amounts of data, allowing you to solve many unconventional problems in a short time [5]. The ease of use of such networks lies in their learnability - there is no need to study various algorithms and hire highly qualified specialists, because the training takes place by examples. But their potential has not been fully revealed, since there are a number of problems that are still being solved at the present time. One of these problems is the insufficient signal transmission rate within the neural network, since the hardware component is weak. It all depends on whether data can be transmitted by computers at a speed close to the speed of human thought [6]. In the near future, these issues will be resolved, and the development of artificial neural networks will move to a new level.

The above is only a small fraction of the whole variety of applications or already use in the field of neural networks, and how many are still in the development stage or plans. Thanks to neural networks, since 2011, the annual volume of investments in the field of AI has grown 15 times, but this is only the very beginning, if you look at the number of startups that are developing in this area [11], there are already tens of thousands of them and, according to analysts, hundreds of them will cost hundreds billions of dollars in just a few years. Already today, only one face recognition market is valued at $\$ 3$ billion and this is only one direction of neural networks. Such rapid development brings improvement in many areas of human life, facilitation of routine work, but at the same time there is a danger of cutting a large number of jobs, and sometimes the complete elimination of an entire profession, because the network will do it faster, better and cheaper. People will have to look for new approaches to accomplishing their tasks, someone will get new work tools that open up new horizons. The whole world and life in it will completely change.

\section{REFERENCES}

1. Hertz J., A. Krogh and R.G. Palmer. Introduction to the Theory of Neural Computation, Reading MA: Addison-Wesley, 1991.

2. Rumelhart D.E. and D. Zipser. "Feature discovery by competitive learning", Cognitive science, 1985, vol. 9, p. 75-112.

3. Bragin A.V., Miroshnichenko V.V., Orlova E.S. Creation of an automated computer system for informational support of a dentist // Problems of dentistry. 2011. No. 4. S. 64-67.

4. Budaeva A.A. Optimization of technologies for multi-criteria ranking of objects // In the book: Operator theory, complex analysis and mathematical modeling, abstracts of the international scientific conference. Southern Mathematical Institute of the Vladikavkaz Scientific Center of the Russian Academy of Sciences and the Government of the Republic of North OssetiaAlania. 2014.S. 166-167.

5. Klepikov A.V., Klyukanov A.V. Virtual excursions // Wagons and wagon facilities. 2014. No. 1 (37). S. 38-39. 
6. Osipov G.S. Optimization of single-channel queuing systems with an unlimited queue // Bulletin of Science and Practice. 2016. No. 9 (10). S. 63-71.

7. Sebeshev V.G. Features of work of statically indeterminate systems and regulation of efforts in structures. - Novosibirsk, 2009.-164 p.

8. Khachaturova K.R. Information technology as a means of development of creative abilities of primary school pupils in natural science lessons // Global scientific potential. 2015. № 9 (54). $111-113 \mathrm{p}$.

9. Masalovich, A. Neural network - the financier's weapon / A. Masalovich [Electronic resource] / Virtual computer museum. - Access mode: http://www.computermuseum.ru/histussr/neiro_net.htm (date accessed: 03/20/18).

10. Serdyukov, V. I. The use of artificial intelligence elements to improve the reliability of technical products / V. I. Serdyukov, N. A. Serdyukov, S. I. Shishkina // Bulletin of mechanical engineering. - 2017. - No. 10. - P. 29-32.

11. Serdyukov, V. I. Improving the failure-free operation of products using elements of artificial intelligence / V. I. Serdyukov, N. A. Serdyukov, S. I. Shishkina // Bulletin of the Moscow State Technical University. N.E. Bauman. Series: mechanical engineering. - 2017. - No. 1 (112). - P. $62-72$.

\title{
About authors:
}

Samat B. Mukhanov, Master of Technical science, senior lecturer, Computer Engineering and Information Security, International Information Technology University.

Arman Ye. Alimbekov, Master student in «Data Science», tutor, Mathematical and Computer Modeling Department, International Information Technology University.

Gaukhar S. Marat, Master student in «Data Science», tutor, Mathematical and Computer Modeling Department, International Information Technology University.

Aibanu M. Uatbayeva, Master student in «Information Systems», International Information Technology University.

Assylkhan A. Aldanazar, Master student in «Computer systems and software engineering», International Information Technology University.

\section{UDC 004.896}

\author{
M.A. Mukanova, I.V. Krak, A.A. Kuandykov, A.S. Sagalova, D.A. Baibatyrov \\ International Information Technology University, Almaty, Kazakhstan
USING VISUAL ANALYTICS TO DEVELOP HUMAN AND MACHINE-CENTRIC MODELS: A REVIEW OF APPROACHES AND PROPOSED INFORMATION TECHNOLOGY

\begin{abstract}
The use of a visual analytical system in machine learning is the basis for the integration of human and the use of his intellectual capabilities in the construction of models. At the same time, visual analytics is used to expand human knowledge and is used as a research tool. We investigate the forms and goals of using visual analytics workflow towards the formation of the final product. Workflow is divided into human-oriented and machine-oriented in order to build a model as an information processor and decisionmaking mechanism. Models are built on the basis of the end user, which can be either a machine or a human. The concepts of model building and the role of machines and humans in these processes are investigated. A practical implementation of the classification information technology in the studied concept 'using opposite model' in the machine-oriented visual analytics workflow for using the machine model is proposed.
\end{abstract}

International Journal of Information and Communication Technologies, Vol.1, Issue 3, September, 2020 